\title{
Flavor origin of R-parity
}

\author{
S. Moris: ${ }^{*}$ \\ Institut für Theoretische Physik und Astrophysik, \\ Universität Würzburg, 97074 Würzburg, Germany \\ E. Peinadd \\ INFN, Laboratori Nazionali di Frascati, Via Enrico Fermi 40, I-00044 Frascati, Italy \\ A. Vicent田 \\ Laboratoire de Physique Théorique, CNRS - UMR 8627, \\ Université de Paris-Sud 11, F-91405 Orsay Cedex, France
}

(Dated: October 31, 2018)

\begin{abstract}
Proton stability is guaranteed in the MSSM by assuming a discrete symmetry, R-parity. However, there are additional R-parity conserving higher dimensional operators which violate lepton and baryon numbers and induce fast proton decay. Here we study the possibility that all renormalizable, as well as the most dangerous non-renormalizable, R-parity violating operators are forbidden by a flavor symmetry, providing a common origin for fermion mixing and proton and dark matter stability. We propose a specific model based on the $\Delta(27)$ discrete symmetry.
\end{abstract}

\section{INTRODUCTION}

In July 2012, the ATLAS [1] and CMS [2] collaborations announced the discovery of a new boson, with a mass in the $126 \mathrm{GeV}$ ballpark. Although detailed studies of its properties need to be done in order to confirm its identity, one is tempted to interpret this new particle in terms of the long-awaited Higgs boson. In any case, this constitutes a decisive breakthrough in high energy physics that, once correctly understood, will surely shed light on the dynamics of the electroweak scale. The discovery of the Higgs boson, and the measurement of its mass, reminds us a longstanding theoretical problem in particle physics: the famous hierarchy problem [3]. This can be expressed as the high sensitivity that fundamental scalars have to physics at high energies. Unless one accepts a very precise fine-tuning of the parameters of the theory, the Higgs mass is naturally pushed to those high energies, distabilizing the electroweak scale. Supersymmetry (SUSY) is one of the most popular solutions to the hierarchy problem. If the SUSY breaking scale is low (not far from the $\mathrm{TeV}$ scale), the electroweak scale is kept under control by virtue of the cancellations between bosonic and fermionic contributions to the Higgs mass.

When constructing a supersymmetric model one finds new gauge and SUSY invariant renormalizable interactions, not present in the standard model, that lead to lepton (L) and baryon (B) number violation. With the particle content of the Minimal Supersymmetric Standard Model (MSSM), these are

$$
\left[L H_{u}\right]_{F}, \quad[L L e]_{F}, \quad[L Q d]_{F}, \quad[u d d]_{F},
$$

where $F$ stands for F-terms. If they were simultaneously present in the lagrangian, the proton would have a fast decay rate unless very small coefficients are introduced. For that reason, one usually introduces a discrete symmetry called R-parity, $R_{p}=(-1)^{3(B-L)+2 s}$ (where $s$ is the spin of the particle), that forbids the $\mathrm{L}$ and $\mathrm{B}$ violating terms shown above [4]. The conservation of R-parity has very relevant phenomenological implications. This discrete symmetry stabilizes the lightest supersymmetric particle (LSP). As a consequence of that, supersymmetric events at colliders contain large amounts of missing energy in the final state. Furthermore, if neutral, the LSP would be the perfect example of a Weakly Interacting Massive Particle (WIMP) and a good dark matter candidate. R-parity has, however, some drawbacks. First of all, R-parity is introduced by hand in the MSSM, without a theoretical explanation for its origin. And second, there are additional non-renormalizable interactions which, even though they break lepton and/or baryon numbers, are perfectly allowed by R-parity. Even if these operators are generated at the Planck scale, they would lead to unnacceptably fast proton decay unless their coefficients are tiny [5, 6].

\footnotetext{
*Electronic address: stefano.morisi@gmail.com

${ }^{\dagger}$ Electronic address: epeinado@lnf.infn.it

${ }^{\ddagger}$ Electronic address: avelino.vicente@th.u-psud.fr
} 


\begin{tabular}{|c|c|c|c|c|c|c|c|}
\hline & $\hat{L}$ & $\hat{e}$ & $\hat{H}_{d}$ & $\hat{H}_{u}$ & $\hat{Q}$ & $\hat{d}$ & $\hat{u}$ \\
\hline$\Delta(27)$ & $\mathbf{3}$ & $\mathbf{1}_{\mathbf{1}, \mathbf{2}, \mathbf{3}}$ & $\mathbf{3}^{*}$ & $\mathbf{3}$ & $\mathbf{3}$ & $\mathbf{1}_{\mathbf{1}, \mathbf{2}, \mathbf{3}}$ & $\mathbf{3}$ \\
\hline
\end{tabular}

TABLE I: $\Delta(27)$ charges of the MSSM superfields.

Many theoretical ideas have been proposed in order to explain the origin of R-parity. Most of them consider Rparity as a remnant after the breaking of a larger symmetry group, see for example [7]. However, in most cases there is no explanation for the suppression of the higher-dimensional operators. On the other hand, the origin of fermion masses hierarchies and mixings, the so-called flavor problem, is another long-standing mystery in particle physics. One interesting possibility to address the flavor problem is the introduction of an horizontal symmetry between the three generations of fermions. The flavor symmetry, which can be either continuous or discrete, imposes some structures on the Yukawa couplings.

In this paper we propose that R-parity may be a consequence of the flavor symmetry. In this case, quark and lepton mixings, as well as the stability of proton and dark matter, can be explained in a common framework. We introduce a flavor model where all renormalizable as well as dimension five L or B violating operators are forbidden by the non-abelian flavor symmetry $\Delta(27)$. Charged femion masses and mixings can be fitted to their observed values. Since the Weinberg operator is also forbidden by the flavor symmetry, one is forced to go beyond minimal models if neutrino masses and mixings are to be explained. This turns out be a non-trivial task due to (1) the restrictions imposed by $\Delta(27)$, and (2) our main motivation of not inducing R-parity violating terms. We find that this can be achieved by extending the lepton sector without inducing dangerous dimension five operators. Neutrino mass arises from a variation of the inverse seesaw mechanism [8], called inverse type-III seesaw mechanism [9], that provides a correct description of neutrino mixings.

The rest of the paper is organized as follows: we present the model and main idea in section II how the introduction of a $\Delta(27)$ flavor symmetry can automatically lead to R-parity conservation and proton stability. Afterwards, we discuss the flavor structure of the resulting quark and lepton sectors in sections III and IV] respectively. In the latter case we extend the original model in order to account for neutrino masses and show how the resulting neutrino mixing pattern can accommodate the data coming from oscillation experiments. Finally, we conclude with a short summary and a brief discussion in section $\mathrm{V}$

\section{THE MODEL: R-PARITY AND PROTON STABILITY FROM $\Delta(27)$}

Let us consider the MSSM extended by a $\Delta(27)$ flavor symmetry, see for instance [10]. This discrete group is a subgroup of $S U(3)$ (for a classification see [11]) that belongs to the series $\Delta\left(3 n^{2}\right)$. It has 11 irreducible representations, namely two triplets $\mathbf{3}, \mathbf{3}^{*}$ and 9 singlets $\mathbf{1}_{\mathbf{i}}$. The product rules for the triplet representations are $\mathbf{3} \times \mathbf{3}^{*}=\sum_{i=1}^{9} \mathbf{1}_{\mathbf{i}}$ and $\mathbf{3} \times \mathbf{3}=\mathbf{3}^{*}+\mathbf{3}^{*}+\mathbf{3}^{*}$. From these rules, it is clear that the product $\mathbf{3} \times \mathbf{3} \times \mathbf{3}$ is invariant under $\Delta(27)$ whereas $\mathbf{3} \times \mathbf{3} \times \mathbf{3}^{*}$ is not. We assign $\Delta(27)$ representations to the MSSM particle content as shown in Table I. It is straightforward to check that this assignment forbids all the aforementioned R-parity violating couplings in Eq. (1). Therefore, R-parity results as an accidental symmetry originated by the underlying flavor symmetry of the model.

The superpotential invariant under $\Delta(27)$ is given by

$$
\mathcal{W}_{\mathrm{MSSM}}=Y_{u} \hat{Q} \hat{H}_{u} \hat{u}+Y_{d} \hat{Q} \hat{H}_{d} \hat{d}+Y_{l} \hat{L} \hat{H}_{d} \hat{e}+\mu \hat{H}_{u} \hat{H}_{d}
$$

here we have omit the $\Delta(27)$ contractions for simplicity. Note, however, that in the product $\mathbf{3} \times \mathbf{3}$ there are three different contractions in the $\mathbf{3}^{*}$ representation. Therefore, each Yukawa coupling in Eq. (2) should be understood as three different parameters, accounting for the three possible $\Delta(27)$ invariant products. We denote them as $Y_{u, d, l}^{1,2,3}$.

Furthermore, the flavor symmetry also forbids the most dangerous non-renormalizable operators (those with lower 
dimensions, $d=5$ ) that break L or B numbers. These $\operatorname{are}^{1}[12]$

$$
\begin{aligned}
\mathcal{O}_{1}^{(5)} & =[Q Q Q L]_{F} & \mathcal{O}_{2}^{(5)} & =[\text { uude }]_{F} \\
\mathcal{O}_{3}^{(5)} & =\left[Q Q Q H_{d}\right]_{F} & \mathcal{O}_{4}^{(5)} & =\left[Q u e H_{d}\right]_{F} \\
\mathcal{O}_{5}^{(5)} & =\left[L L H_{u} H_{u}\right]_{F} & \mathcal{O}_{6}^{(5)} & =\left[L H_{d} H_{u} H_{u}\right]_{F} \\
\mathcal{O}_{7}^{(5)} & =\left[H_{u} H_{u} e^{*}\right]_{D} & \mathcal{O}_{8}^{(5)} & =\left[H_{u}^{*} H_{d} e\right]_{D} \\
\mathcal{O}_{9}^{(5)} & =\left[Q u L^{*}\right]_{D} & \mathcal{O}_{10}^{(5)} & =\left[u d^{*} e\right]_{D}
\end{aligned}
$$

Here, we include F-terms that may be present in the superpotential and D-terms which may be present in the Kähler potential. They are denoted with the subscripts $F$ and $D$, respectively. These operators appear in the lagrangian with a mass suppression $1 / \Lambda$, where $\Lambda$ is the energy scale associated to the $\mathrm{L}$ and/or B number violating physics beyond the MSSM. Even if this scale is taken as large as the Planck scale, these dangerous operators would lead to too fast proton decay if the corresponding coefficients are of order one. Since all these operators are forbidden by $\Delta(27)$, we conclude that, regarding proton stability, the flavor symmetry makes a better job than R-parity ${ }^{2}$.

One could similarly list all dimension six $(d=6)$ non-renormalizable operators that induce lepton and/or baryon number violation [12]. This list is, of course, much longer. Although $\Delta(27)$ forbids many of them, some are allowed. A simple example is $\mathcal{O}^{(6)}=\left[u d d L H_{u}\right]_{F}$, which breaks both lepton and baryon numbers. These operators have a mass suppression $1 / \Lambda^{2}$ and thus they are less dangerous than the $d=5$ ones, only requiring relatively small coefficients if $\Lambda \sim m_{G U T}=2 \cdot 10^{16} \mathrm{GeV}[12]$.

In conclusion, the flavor model given by the $\Delta(27)$ charges in Table 1 stabilizes the proton (including all dimension 5 dangerous operators) and leads to automatic R-parity conservation. Furthermore, the $\Delta(27)$ symmetry allows the usual three fermion Yukawa couplings, as well as the $\mu$ term. Therefore, we recover the usual MSSM (with the restrictions imposed by the flavor symmetry) with a clear improvement regarding proton stability. The next check that we need to make is the viability of the model regarding fermion masses and mixings.

\section{THE QUARK SECTOR}

In order to get the structure of the fermion mass matrices, we give here the relevant contractions of the $\Delta(27)$ group. As mentioned before, the product rules for triplet representations are $\mathbf{3} \times \mathbf{3}^{*}=\sum_{i=1}^{9}\left(\mathbf{3} \times \mathbf{3}^{*}\right)_{i} \equiv \sum_{i=1}^{9} \mathbf{1}_{\mathbf{i}}$. If one denotes the triplet representations as $\mathbf{3}=\left(a_{1}, a_{2}, a_{3}\right)$ and $\mathbf{3}^{*}=\left(b_{1}, b_{2}, b_{3}\right)$, the explicit singlet contractions are given by

$$
\begin{aligned}
& \mathbf{1}_{\mathbf{1}}=a_{1} b_{1}+a_{2} b_{2}+a_{3} b_{3}, \\
& \mathbf{1}_{\mathbf{2}}=a_{1} b_{1}+a_{2} b_{2} \omega+a_{3} b_{3} \omega^{2}, \\
& \mathbf{1}_{\mathbf{3}}=a_{1} b_{1}+a_{2} b_{2} \omega^{2}+a_{3} b_{3} \omega, \\
& \mathbf{1}_{\mathbf{4}}=a_{1} b_{2}+a_{2} b_{3}+a_{3} b_{1}, \\
& \mathbf{1}_{\mathbf{5}}=a_{1} b_{2}+a_{2} b_{3} \omega+a_{3} b_{1} \omega^{2}, \\
& \mathbf{1}_{\mathbf{6}}=a_{1} b_{2}+a_{2} b_{3} \omega^{2}+a_{3} b_{1} \omega, \\
& \mathbf{1}_{\mathbf{7}}=a_{2} b_{1}+a_{3} b_{2}+a_{1} b_{3}, \\
& \mathbf{1}_{\mathbf{8}}=a_{2} b_{1}+a_{3} b_{2} \omega+a_{1} b_{3} \omega^{2}, \\
& \mathbf{1}_{\mathbf{9}}=a_{2} b_{1}+a_{3} b_{2} \omega^{2}+a_{1} b_{3} \omega,
\end{aligned}
$$

\footnotetext{
${ }^{1}$ In general, some of these operators will not be generated in a given model. For example, the operator $\mathcal{O}_{7}^{(5)}$ will be absent if there is only one $\hat{H}_{u}$ superfield since the antisymmetric $S U(2)$ contraction would vanish exactly.

2 The model in Ref. [13] also leads to R-parity as a by-product of a flavor symmetry. However, some $d=5$ operators are allowed by their symmetry. We also note that other alternatives to R-parity can also forbid (or strongly suppress) higher-dimensional operators with lepton and/or baryon number violation, see for example [14].
} 
with $\omega^{3}=1$. Similarly, one can obtain the product $\mathbf{3} \times \mathbf{3}=\mathbf{3}^{*}+\mathbf{3}^{*}+\mathbf{3}^{*}$. If $\mathbf{3}=\left(a_{1}, a_{2}, a_{3}\right)$ and $\mathbf{3}=\left(b_{1}, b_{2}, b_{3}\right)$, the corresponding contractions are

$$
\left(\begin{array}{l}
a_{1} b_{1} \\
a_{2} b_{2} \\
a_{3} b_{3}
\end{array}\right) \oplus\left(\begin{array}{l}
a_{2} b_{3} \\
a_{3} b_{1} \\
a_{1} b_{2}
\end{array}\right) \oplus\left(\begin{array}{l}
a_{3} b_{2} \\
a_{1} b_{3} \\
a_{2} b_{1}
\end{array}\right) .
$$

The Yukawa term for the $d$ quarks (as well as for the charged leptons) involves the $\Delta(27)$ product $\mathbf{3} \times \mathbf{3}^{*} \times \mathbf{1}_{\mathbf{i}}$ where the singlets are $1_{1}, 1_{2}$ and $1_{3}$, respectively. The mass matrix reads

$$
M_{d} \sim\left(\begin{array}{ccc}
Y_{d}^{1}\left\langle H_{d}^{1}\right\rangle & Y_{d}^{2}\left\langle H_{d}^{1}\right\rangle & Y_{d}^{3}\left\langle H_{d}^{1}\right\rangle \\
Y_{d}^{1}\left\langle H_{d}^{2}\right\rangle & \omega Y_{d}^{2}\left\langle H_{d}^{2}\right\rangle & \omega^{2} Y_{d}^{3}\left\langle H_{d}^{2}\right\rangle \\
Y_{d}^{1}\left\langle H_{d}^{3}\right\rangle & \omega^{2} Y_{d}^{2}\left\langle H_{d}^{3}\right\rangle & \omega Y_{d}^{3}\left\langle H_{d}^{3}\right\rangle
\end{array}\right)
$$

The $u$ quark mass matrix comes from the $\Delta(27)$ product $\mathbf{3} \times \mathbf{3} \times \mathbf{3}$, which leads to

$$
M_{u} \sim\left(\begin{array}{ccc}
Y_{u}^{1}\left\langle H_{u}^{1}\right\rangle & Y_{u}^{2}\left\langle H_{u}^{2}\right\rangle & Y_{u}^{3}\left\langle H_{u}^{1}\right\rangle \\
Y_{u}^{3}\left\langle H_{u}^{2}\right\rangle & Y_{u}^{1}\left\langle H_{u}^{2}\right\rangle & Y_{u}^{2}\left\langle H_{u}^{3}\right\rangle \\
Y_{u}^{2}\left\langle H_{u}^{1}\right\rangle & Y_{u}^{3}\left\langle H_{u}^{3}\right\rangle & Y_{u}^{1}\left\langle H_{u}^{3}\right\rangle
\end{array}\right)
$$

It is not difficult to show that by taking ${ }^{3}$ the vaccum expectation value $(\mathrm{VEV})$ alignment $\left\langle H_{u, d}^{0}\right\rangle \sim(1,1,1)$, which breaks $\Delta(27)$ into a $Z_{3}$ subgroup, all the charged fermion mass matrices are diagonalized on the left by the same unitary matrix, the so-called magic matrix $U_{\omega}$, defined as

$$
U_{\omega}=\frac{1}{\sqrt{3}}\left(\begin{array}{ccc}
1 & 1 & 1 \\
1 & \omega & \omega^{2} \\
1 & \omega^{2} & \omega
\end{array}\right)
$$

namely

$$
U_{\omega}^{\dagger} M_{u} \cdot M_{u}^{\dagger} U_{\omega}=D_{u}^{2}, \quad U_{\omega}^{\dagger} M_{d} \cdot M_{d}^{\dagger} U_{\omega}=D_{d}^{2}
$$

where $D_{u, d}$ are diagonal matrices whose entries are functions of the Yukawa couplings for each sector. Then, each charged fermion mass matrix in Eqs. (4) and (5) can be fitted to reproduce the corresponding three fermion masses and the CKM mixing matrix, $V_{C K M}=U_{u}^{\dagger} U_{d}$, turns out to be proportional to the identity, since $U_{u}=U_{d}=U_{\omega}$. This is usually regarded as a good starting point when building a model. As next step one can consider a completely broken $\Delta(27)$, breaking the alignment $\left\langle H_{u, d}^{0}\right\rangle \sim(1,1,1)$, and leading to a CKM that deviates from the identity. Similarly, the CKM matrix can also be generated at the loop level from the flavor structure associated with the SUSY breaking terms [16]. Other possibilities to generate the CKM mixing could be by means of adding extra mirror quarks, scalar-mediated interactions [17] or by using different singlets of $\Delta(27)$ [18].

We now turn to the next section, where we discuss extensions of the lepton sector to generate non-zero neutrino masses. Although one may expect to find many possible directions, we will find that this task turns out to be far from trivial due to the restrictions imposed by $\Delta(27)$.

\section{THE LEPTON SECTOR}

\section{A. Charged lepton}

The charged lepton mass matrix has the same structure as the $d$ quark mass matrix, that is

$$
M_{l} \sim\left(\begin{array}{ccc}
Y_{l}^{1}\left\langle H_{d}^{1}\right\rangle & Y_{l}^{2}\left\langle H_{d}^{1}\right\rangle & Y_{l}^{3}\left\langle H_{d}^{1}\right\rangle \\
Y_{l}^{1}\left\langle H_{d}^{2}\right\rangle & \omega Y_{l}^{2}\left\langle H_{d}^{2}\right\rangle & \omega^{2} Y_{l}^{3}\left\langle H_{d}^{2}\right\rangle \\
Y_{l}^{1}\left\langle H_{d}^{3}\right\rangle & \omega^{2} Y_{l}^{2}\left\langle H_{d}^{3}\right\rangle & \omega Y_{l}^{3}\left\langle H_{d}^{3}\right\rangle
\end{array}\right),
$$

\footnotetext{
3 The alignment $\left\langle H_{u, d}^{0}\right\rangle \sim(1,1,1)$ will be assumed as a starting point in the derivation of the fermion mixing matrices. Although we do not address its origin, we note that this alignment is natural in models based on the $\Delta(27)$ discrete symmetry [15]. Nevertheless, small deviations from this particular alignment do not change our main conclusions and would be welcome in order to obtain a realistic CKM matrix (see below).
} 


\begin{tabular}{|c|c|c|c|}
\hline & $\hat{\Sigma}_{1}$ & $\hat{\Sigma}_{2}$ & $\hat{S}$ \\
\hline$\Delta(27)$ & $\mathbf{3}$ & $\mathbf{3}^{*}$ & $\mathbf{3}^{*}$ \\
\hline
\end{tabular}

TABLE II: Charge assignment of the additional superfields in the extended model for neutrino masses. The superfields $\hat{\Sigma}_{1,2}$ are triplets of $S U(2)_{L}$ with $Y=0$ and $\hat{S}$ is a neutral $S U(2)_{L}$ singlet.

therefore if the vaccum expectation value $(\mathrm{VEV})$ alignment $\left\langle H_{u, d}^{0}\right\rangle \sim(1,1,1)$, this matrix is also diagonalized by the $U_{\omega}$ matrix given in Eq. (7).

\section{B. Neutrino masses - inverse type-III seesaw}

So far, we have only discussed the viability of the framework. Unfortunately, the model resulting from the addition of the $\Delta(27)$ discrete symmetry is just the MSSM, with some restrictions in the parameters ${ }^{4}$. Therefore, we do not expect any new collider signature that is not present in the canonical MSSM. In order to find new predictions one needs to extend the model in order to account for neutrino masses. The new structures must preserve $\Delta(27)$ as well, and this leads to interesting consequences.

As shown above, the flavor symmetry forbids the Weinberg operator, $\mathcal{O}_{5}^{(5)}=\left[L L H_{u} H_{u}\right]_{F}$. Therefore, in order to generate neutrino masses one is forced to go beyond minimal models and consider higher dimensional operators.

To the particle content in Table I, we add the superfields in Table【. The singlet $\hat{S}$ should not be confused with the NMSSM singlet superfield. In fact, note that the $\hat{S} \hat{H}_{u} \hat{H}_{d}$ superpotential term is forbidden by $\Delta(27)$. Besides $\mathcal{W}_{M S S M}$ defined in Eq. (2) the superpotential contains

$$
\mathcal{W} \supset Y_{\Sigma} \hat{L} \hat{H}_{u} \hat{\Sigma}_{1}+M \hat{\Sigma}_{1} \hat{\Sigma}_{2}+\lambda \hat{S} \hat{\Sigma}_{2} \hat{\Sigma}_{2}+\kappa_{S} \hat{S}^{3}
$$

Other superpotential terms are forbidden by the gauge and flavor symmetries ${ }^{5}$. When the scalar component of $\hat{S}$ gets a VEV, an effective Majorana mass for the $\Sigma_{2}$ triplet (the fermionic component of the $\hat{\Sigma}_{2}$ superfield) is generated. This leads to an inverse seesaw mechanism [8] induced by $S U(2)_{L}$ triplets (for other realizations of the inverse type-III seesaw see [9]). In the basis $\psi^{T}=\left(\nu, \Sigma_{1}^{0}, \Sigma_{2}^{0}\right)$, we obtain the $9 \times 9$ mass matrix for the neutral fermions

$$
M_{\nu}=\left(\begin{array}{ccc}
0 & Y_{\Sigma}\left\langle H_{u}\right\rangle & 0 \\
Y_{\Sigma}^{T}\left\langle H_{u}\right\rangle & 0 & M \\
0 & M^{T} & \lambda\langle S\rangle
\end{array}\right),
$$

which, assuming $\lambda v_{S} \ll Y_{\Sigma} v_{u} \ll M$, leads to

$$
m_{\nu}=v_{u}^{2} v_{S} Y_{\Sigma}\left(M^{T}\right)^{-1} \lambda M^{-1} Y_{\Sigma}^{T}
$$

where $v_{u}=\left\langle H_{u}^{0}\right\rangle$ and $v_{S}=\langle S\rangle$. In fact, it is worth emphasizing some advantages that our model has with respect to the conventional inverse seesaw. Typically, in the context of inverse seesaw models it is quite difficult to forbid the $\Sigma_{1}^{0} \Sigma_{1}^{0}$ mass term after ones allows for lepton number violation. Here its absence is a direct consequence of the $\Delta(27)$ symmetry. Moreover, in order to have new physics at the $\mathrm{TeV}$ scale, that is $M \sim \mathrm{TeV}$, and $\mathcal{O}(1)$ Yukawa couplings, the parameter $\mu_{S}=\lambda v_{S}$ must be of the order of $10 \mathrm{eV}$. In the original inverse seesaw mechanism the $\mu_{S}$ parameter is expected to be naturally small in the 't Hooft sence since the limit $\mu_{S} \rightarrow 0$ enhances the symmetry of the lagrangian (lepton number is recovered). Although this is not the case here, $\mu_{S}$ can be suppressed by means of the VEV of the scalar $S$. Moreover, several mechanisms to obtain dynamically a small $\mu_{S}$ parameter are known in the literature [19]. For these reasons, we consider a small $\mu_{S}$ parameter a natural choice.

As mentioned above, the flavor symmetry forbids the Weinberg operator. In fact, in this model neutrino masses are generated due to the effective operator

$$
\mathcal{O}^{(6)}=\left[L L H_{u} H_{u} S\right]_{F},
$$

\footnotetext{
${ }^{4}$ To be precise, the only difference with the canonical MSSM is the existence of three pairs of Higgs doublets, as discussed below.

${ }^{5}$ We note that if the $\hat{\Sigma}_{1,2}$ were singlets under $S U(2)_{L}$, superpotential terms $\kappa_{\Sigma_{i}} \hat{\Sigma}_{i}^{3}$ would be allowed, thus breaking R-parity explicitly.
} 


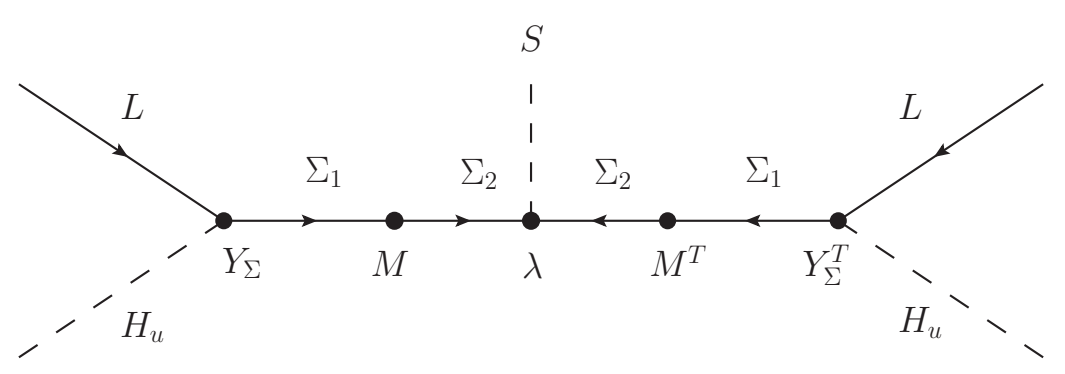

FIG. 1: $\mathcal{O}^{(6)}=\left[L L H_{u} H_{u} S\right]_{F}$ realization.

which is obtained after integrating out the $\hat{\Sigma}_{1,2}$ superfields, as depicted in Figure (11). Therefore, the proposed model can be seen as a concrete renormalizable realization of this operator, which is the minimal implementation of Majorana neutrino masses after the Weinberg operator is forbidden.

Lepton number is explicitly broken by the trilinear superpotential term $\kappa_{S} \hat{S}^{3}$ and thus no majoron, the Goldstone boson associated to the spontaneous breaking of $U(1)_{L}$, appears in the spectrum. However, R-parity remains unbroken after the addition of this term, since $S$ carries two units of lepton number. One may also wonder about the appearance of new unwanted interactions after the introduction of the $\hat{S}$ superfield. It is straightforward to check that no L or $\mathrm{B}$ violating renormalizable operators are allowed, apart from the aforementioned $\kappa_{S} \hat{S}^{3}$ term. Regarding higher dimensional operators, we note that the operator $\mathcal{O}^{(5)}=[u d d S]_{F}$ is the only dimension 5 operator (with $\mathrm{L}$ or $\mathrm{B}$ violation) that is allowed by the symmetries. However, it cannot be generated at tree-level, since $\hat{S}$ does not couple to the quark superfields, and only some specific high-energy completions of our model would generate it at the (less dangerous) loop level.

\section{Neutrino mixing pattern}

Let us now discuss the resulting neutrino mixing pattern. Using the $\Delta(27)$ contraction rules, one finds that the mass matrix $M$ is proportional to the identity matrix. The Dirac neutrino mass matrix $m_{D}=Y_{\Sigma}\left\langle H_{u}^{0}\right\rangle$ and the matrix $\mu_{S}=\lambda\langle S\rangle$ have the structures

$$
m_{D}=\left(\begin{array}{ccc}
\alpha & \beta & \gamma \\
\gamma & \alpha & \beta \\
\beta & \gamma & \alpha
\end{array}\right), \mu_{S}=\left(\begin{array}{ccc}
\alpha^{\prime} & \beta^{\prime} & \beta^{\prime} \\
\beta^{\prime} & \alpha^{\prime} & \beta^{\prime} \\
\beta^{\prime} & \beta^{\prime} & \alpha^{\prime}
\end{array}\right)
$$

when all the scalar fields take VEV in the $(1,1,1)$ direction. In this case, the charged lepton mass matrix ${ }^{6}$ is diagonalized by the magic matrix $U_{\omega}$, see Eq. (7). One can now perform a $U_{\omega}$ rotation in order to go to the basis where the charged lepton mass matrix is diagonal. In this basis, the neutrino mass matrix can be written as

$$
\tilde{m}_{\nu} \sim U_{\omega} m_{\nu} U_{\omega}^{T} \sim\left(\begin{array}{ccc}
a & 0 & 0 \\
0 & 0 & b \\
0 & b & 0
\end{array}\right)
$$

where the parameters $a$ and $b$ are functions of $\alpha, \beta, \gamma, \alpha^{\prime}, \beta^{\prime}, \gamma^{\prime}$. In general $a \neq b$. By setting $a=b$ we recover the result of the Babu-Ma-Valle model [20]. In this limit, the mass matrix in Eq. (14) gives maximal atmospheric angle and degenerate neutrino mass spectrum, while the solar and reactor mixing angles are zero. As observed in [20], Eq. (14) is corrected by wave-function renormalizations of $\nu_{e}, \nu_{\mu}$, and $\nu_{\tau}$, as well as the corresponding vertex renormalizations, lifting the neutrino degeneracy and the solar/reactor mixing angles. The resulting neutrino mass matrix can be written as

$$
\tilde{m}_{\nu}^{1-\text { loop }} \sim\left(\begin{array}{ccc}
a\left(1+2 \delta_{e e}\right) & a \delta_{e \mu}+b \delta_{e \tau}^{*} & b \delta_{e \mu}^{*}+a \delta_{e \tau} \\
& 2 b \delta_{\mu \tau}^{*} & b\left(1+\delta_{\mu \mu}+\delta_{\tau \tau}\right) \\
& & 2 b \delta_{\mu \tau}
\end{array}\right)
$$

\footnotetext{
${ }^{6}$ After electroweak symmetry breaking, charged leptons mix with the charged components of the $\Sigma$ fermions. This leads, however, to numerically irrelevant corrections in the leptonic mixing matrix.
} 
where $\delta_{i j}$ parametrize the radiative corrections. When $a=b$ (as in [20]) and assuming real $\delta_{i j}$ corrections, the resulting neutrino mass matrix is $\mu-\tau$ symmetric giving maximal atmospherc angle and zero $\theta_{13}$ reactor angle. The solar angle is a free parameter and can be fitted. If the corrections are allowed to be complex, a non-zero value for $\theta_{13}$ can be obtained. In this case, $\mathrm{CP}$ violation is predicted to be maximal [20]. Regarding the nature of the corrections, these come from flavor mixing in the slepton/sneutrino sector. A detailed study can be found in [21]. Note that our model has more freedom since $a \neq b$ in general. This can be used to relax some of the restrictions in the parameter space. Nevertheless, large $\tilde{\mu}-\tilde{\tau}$ mixing is necessary, typically predicting $\operatorname{Br}(\tau \rightarrow \mu \gamma)$ close to its experimental limit. Furthermore, in the SUSY inverse seesaw one expects large Z-penguin contributions in lepton flavor violating processes [22]. Therefore, observables such as $\operatorname{Br}(\tau \rightarrow \mu \ell \ell)$, with $\ell=e, \mu$, are also expected to set important constraits on the SUSY parameter space. Finally, violation of lepton flavor universality in observables such as $R_{K}$ and $R_{\pi}$ is also an important test of the model [23].

\section{SUMMARY AND DISCUSSION}

In summary, we have proposed a supersymmetric flavor model based on the $\Delta(27)$ symmetry. All lepton and/or baryon number violating operators of dimension 4 and 5 are forbidden by the flavor symmetry, thus providing a single explanation for the proton and dark matter stabilities ${ }^{7}$. The extension to account for neutrino masses and mixings turned out to be non-trivial due to the restrictions imposed by the $\Delta(27)$ symmetry. In fact, naive extensions of the leptonic sector typically spoil the nice features of the original model and depart from the main motivations for this work. We found that one can indeed generate neutrino masses by means of an inverse type-III seesaw, while preserving R-parity and keeping the proton stable. The phenomenology of the complete model has been briefly discussed. In particular, the observed neutrino mixing pattern (with a large reactor angle) can be well accommodated.

Before concluding the paper we would like to comment on a difference that our setup has with respect to the canonical MSSM: the existence of three Higgs doublets. This feature, the replication of the minimal Higgs sector, is shared by all flavor models where the usual Higgs doublet is promoted to a multiplet of the flavor group. This typically implies very complicated scalar potentials, whose minimization leads to viable vacua (from a phenomenological point of view) thanks to the soft SUSY breaking terms. An example in the context of the $A_{4}$ symmetry can be found in Ref. 25]. Although the model under consideration here involves a different flavor symmetry, similar results are expected. A detailed minimization of the potential and determination of the scalar spectrum is clearly beyond the scope of the paper. Furthermore, if the new scalar/pseudoscalar states can be made relatively light, one may expect sizable Higgs-mediated contributions to flavor processes such as $\tau \rightarrow 3 \mu, B_{d, s} \rightarrow \ell_{i} \ell_{j}$ or $\tau \rightarrow \mu P$ (where $P$ denotes a neutral pseudoscalar meson $)^{8}$ [26].

The extended Higgs sector provides additional freedom to accommodate the observed $126 \mathrm{GeV}$ Higgs-like resonance found by ATLAS and CMS. In fact, one can easily find regions in parameter space very similar to the so-called decoupling limit of the MSSM, where the lightest scalar particle behaves as the SM Higgs boson. We note that the non-standard corrections coming from the triplets are typically very small due to the relatively small Yukawa couplings (see for example Ref. [28]).

Although most of the phenomenological features of our setup can be found in other flavor models, their combination is quite distinctive. In particular, the presence of the light $\Sigma_{1,2} S U(2)_{L}$ fermion triplet leads to a very rich collider phenomenology, since it can be pair produced at the LHC due to its gauge interactions. This possibility, not present in singlet extensions of the MSSM, may lead to very clear signatures with additional leptons and/or lepton flavor violation in the $\Sigma_{1,2}$ decays [27]. It is well-known that the assumption of R-parity in the MSSM serves to stabilize the LSP. Here we obtain the same result, R-parity, without assuming it. Therefore, the usual MSSM candidate, a neutralino LSP, can play the role of the dark matter of the universe.

\section{Acknowledgements}

The authors are grateful to Werner Porod for useful comments on the manuscript. This work was supported by grants FPA2011-22975, MULTIDARK CSD2009-00064, Prometeo/2009/091, EU ITN UNILHC PITN-GA-2009237920, S.M has been also supported by DFG grant WI 2639/4-1. A.V. acknowledges support by the ANR project

\footnotetext{
${ }^{7}$ For dark matter stability from the spontaneous breaking of a non-Abelian discrete flavor symmetry see [24].

8 The magnitude of these contributions will depend, of course, on the value of $\tan \beta$.
} 
CPV-LFV-LHC NT09-508531.

[1] F. Gianotti, for the ATLAS Collaboration, CERN Seminar, July 4th, 2012.

[2] J. Incandela, for the CMS Collaboration, CERN Seminar, July 4th, 2012.

[3] E. Gildener, Phys. Rev. D 14 (1976) 1667.

[4] G. R. Farrar and P. Fayet, Phys. Lett. B 76 (1978) 575.

[5] S. Weinberg, Phys. Rev. D 26 (1982) 287; N. Sakai and T. Yanagida, Nucl. Phys. B 197 (1982) 533; L. E. Ibanez and G. G. Ross, Nucl. Phys. B 368 (1992) 3.

[6] P. Nath and P. Fileviez Perez, Phys. Rept. 441 (2007) 191 hep-ph/0601023.

[7] R. N. Mohapatra, Phys. Rev. D 34 (1986) 3457; S. P. Martin, Phys. Rev. D 46 (1992) 2769 hep-ph/9207218.

[8] R. N. Mohapatra and J. W. F. Valle, Phys. Rev. D 34 (1986) 1642.

[9] E. Ma, Mod. Phys. Lett. A 24 (2009) 2491 arXiv:0905.2972 [hep-ph]]; D. Ibanez, S. Morisi and J. W. F. Valle, Phys. Rev. D 80 (2009) 053015 arXiv:0907.3109 [hep-ph]]; F. -X. Josse-Michaux and E. Molinaro, arXiv:1210.7202 [hep-ph].

[10] C. Luhn, S. Nasri and P. Ramond, J. Math. Phys. 48, 073501 (2007) hep-th/0701188]; H. Ishimori, T. Kobayashi, H. Ohki, H. Okada, Y. Shimizu and M. Tanimoto, Lect. Notes Phys. 858 (2012) 1.

[11] H. Ishimori, T. Kobayashi, H. Ohki, Y. Shimizu, H. Okada and M. Tanimoto, Prog. Theor. Phys. Suppl. 183 (2010) 1 arXiv:1003.3552 [hep-th]].

[12] D. Piriz and J. Wudka, Phys. Rev. D 56 (1997) 4170 hep-ph/9707314].

[13] C. D. Carone, L. J. Hall and H. Murayama, Phys. Rev. D 54 (1996) 2328 hep-ph/9602364.

[14] H. K. Dreiner, C. Luhn and M. Thormeier, Phys. Rev. D 73 (2006) 075007 hep-ph/0512163; H. M. Lee, S. Raby, M. Ratz, G. G. Ross, R. Schieren, K. Schmidt-Hoberg and P. K. S. Vaudrevange, Phys. Lett. B 694 (2011) 491 arXiv:1009.0905 [hep-ph]].

[15] I. de Medeiros Varzielas, S. F. King and G. G. Ross, Phys. Lett. B 648 (2007) 201 hep-ph/0607045.

[16] K. S. Babu, B. Dutta and R. N. Mohapatra, Phys. Rev. D 60 (1999) 095004 hep-ph/9812421.

[17] L. Lavoura and H. Kuhbock, Eur. Phys. J. C 55, 303 (2008) arXiv:0711.0670 [hep-ph].

[18] G. Bhattacharyya, I. de Medeiros Varzielas and P. Leser, arXiv:1210.0545 [hep-ph].

[19] E. Ma, Phys. Rev. D 80 (2009) 013013 arXiv:0904.4450 [hep-ph]]; P. S. B. Dev and R. N. Mohapatra, Phys. Rev. D 81 (2010) 013001 arXiv:0910.3924 [hep-ph]]; F. Bazzocchi, Phys. Rev. D 83 (2011) 093009 arXiv:1011.6299 [hep-ph]]; A. G. Dias, C. A. de S.Pires, P. S. Rodrigues da Silva and A. Sampieri, Phys. Rev. D 86 (2012) 035007 arXiv:1206.2590 [hep-ph]].

[20] K. S. Babu, E. Ma and J. W. F. Valle, Phys. Lett. B 552 (2003) 207 hep-ph/0206292.

[21] M. Hirsch, J. C. Romao, S. Skadhauge, J. W. F. Valle and A. Villanova del Moral, Phys. Rev. D 69 (2004) 093006 hep-ph/0312265.

[22] M. Hirsch, F. Staub and A. Vicente, Phys. Rev. D 85 (2012) 113013 arXiv:1202.1825 [hep-ph]; M. Hirsch, W. Porod, L. Reichert and F. Staub, Phys. Rev. D 86 (2012) 093018 arXiv:1206.3516 [hep-ph]]; A. Abada, D. Das, A. Vicente and C. Weiland, JHEP 1209 (2012) 015 [arXiv:1206.6497 [hep-ph]].

[23] A. Abada, D. Das, A. M. Teixeira, A. Vicente and C. Weiland, arXiv:1211.3052 [hep-ph].

[24] M. Hirsch, S. Morisi, E. Peinado and J. W. F. Valle, Phys. Rev. D 82 (2010) 116003 arXiv:1007.0871 [hepph]];M. S. Boucenna, M. Hirsch, S. Morisi, E. Peinado, M. Taoso and J. W. F. Valle, JHEP 1105 (2011) 037 arXiv:1101.2874 [hep-ph]].

[25] F. Bazzocchi, S. Morisi, E. Peinado, J. W. F. Valle and A. Vicente, JHEP 1301 (2013) 033 arXiv:1202.1529 [hep-ph]].

[26] A. Dedes, J. R. Ellis and M. Raidal, Phys. Lett. B 549 (2002) 159 hep-ph/0209207; K. S. Babu and C. Kolda, Phys. Rev. Lett. 89 (2002) 241802 hep-ph/0206310]; A. Abada, D. Das and C. Weiland, JHEP 1203 (2012) 100 arXiv:1111.5836 [hep-ph]].

[27] R. Franceschini, T. Hambye and A. Strumia, Phys. Rev. D 78, 033002 (2008) arXiv:0805.1613 [hep-ph]]; F. del Aguila and J. A. Aguilar-Saavedra, Nucl. Phys. B 813 (2009) 22 arXiv:0808.2468 [hep-ph]]; F. del Aguila and J. A. Aguilar-Saavedra, Phys. Lett. B 672 (2009) 158 [arXiv:0809.2096 [hep-ph]].

[28] A. Elsayed, S. Khalil and S. Moretti, Phys. Lett. B 715 (2012) 208 [arXiv:1106.2130 [hep-ph]]. 